\title{
Liquid biopsy monitoring uncovers acquired RAS-mediated resistance to cetuximab in a substantial proportion of patients with head and neck squamous cell carcinoma
}

\author{
Friederike Braig ${ }^{1, *}$, Minna Voigtlaender ${ }^{1, *}$, Aneta Schieferdecker ${ }^{1}$, Chia-Jung \\ Busch $^{2}$, Simon Laban ${ }^{2,3}$, Tobias Grob ${ }^{4}$, Malte Kriegs ${ }^{5}$, Rainald Knecht ${ }^{2}$, Carsten \\ Bokemeyer $^{1}$ and Mascha Binder ${ }^{1}$ \\ 1 Department of Oncology and Hematology with Sections Bone Marrow Transplant and Pneumology, Hubertus Wald \\ Tumorzentrum / University Cancer Center Hamburg, University Medical Center Hamburg, Hamburg, Germany \\ 2 Department of Otorhinolaryngology, Head and Neck Cancer Center of The University Cancer Center Hamburg, University \\ Medical Center Hamburg, Hamburg, Germany \\ ${ }^{3}$ Department of Otorhinolaryngology and Head and Neck Surgery, Ulm University Medical Center, UIm, Germany \\ ${ }^{4}$ Department of Pathology, University Medical Center Hamburg, Hamburg, Germany \\ ${ }^{5}$ Laboratory for Radiobiology and Experimental Radiooncology, Head and Neck Cancer Center of The University Cancer \\ Center Hamburg, University Medical Center Hamburg, Hamburg, Germany \\ * These authors have contributed equally to this work \\ Correspondence to: Mascha Binder, email: m.binder@uke.de \\ Keywords: head and neck squamous cell carcinoma (HNSCC), epidermal growth factor receptor (EGFR), RAS, resistance, cetux- \\ imab \\ Received: March 25, 2016 \\ Accepted: March 29, 2016 \\ Published: April 22, 2016
}

ABSTRACT

Resistance to epidermal growth factor receptor (EGFR)-targeted therapy is insufficiently understood in head and neck squamous cell carcinoma (HNSCC), entailing the lack of predictive biomarkers.

Here, we studied resistance-mediating EGFR ectodomain and activating RAS mutations by next-generation sequencing (NGS) of cell lines and tumor tissue of cetuximab-naïve patients ( 46 cases, 12 cell lines), as well as liquid biopsies taken during and after cetuximab/platinum/5-fluorouracil treatment ( 20 cases). Tumors of cetuximab-naïve patients were unmutated, except for HRAS mutations in $\mathbf{4 . 3 \%}$ of patients. Liquid biopsies revealed acquired KRAS, NRAS or HRAS mutations in more than one third of patients after cetuximab exposure. $46 \%$ of patients with on-treatment disease progression showed acquired RAS mutations, while no RAS mutations were found in the non-progressive subset of patients, indicating that acquisition of RAS mutant clones correlated significantly with clinical resistance (Chi square $p=0.032$ ). The emergence of mutations preceded clinical progression in half of the patients, with a maximum time from mutation detection to clinical progression of 16 weeks.

RAS mutations account for acquired resistance to EGFR-targeting in a substantial proportion of HNSCC patients, even though these tumors are rarely mutated at baseline. Liquid biopsies may be used for mutational monitoring to guide treatment decisions. 


\section{INTRODUCTION}

Head and neck squamous cell carcinomas (HNSCC) arising in the larynx, pharynx, oral cavity, paranasal sinuses and nasal cavity are among the most common types of cancers, accounting for almost 60,000 newly diagnosed cases and more than 10,000 estimated deaths per year in the United States alone [1]. The prognosis of HNSCC patients with locoregionally advanced tumors is poor as indicated by five year overall survival rates of $40-60 \%$ in recent clinical trials [2-6]. In recurrent and metastatic disease the mean overall survival does not exceed 11 months despite intensive treatment protocols $[7,8]$.

Cetuximab, a monoclonal antibody targeting the extracellular ligand binding domain of the epidermal growth factor receptor (EGFR), is approved for the treatment of locoregionally advanced HNSCC in combination with radiotherapy [9] and for the treatment of recurrent or metastatic disease in combination with platinum-based chemotherapy [7]. However, not all patients treated with cetuximab respond well to therapy due to primary or acquired resistance, limiting significantly the clinical benefit of this drug.

Still, the molecular mechanisms underlying clinical resistance to cetuximab in HNSCC have not yet been elucidated. In metastatic colorectal cancer (mCRC) resistance mechanisms are by far better understood and involve mutations in EGFR downstream signaling molecules such as RAS [10]. Constitutive RAS signaling is mediated by mutations that prevent GTP hydrolysis, thus locking RAS in a permanently active state, independent of EGFR engagement. For this reason, colon tumors harboring activating RAS mutations do not respond to EGFR targeting and mutational screening is therefore routinely used for patient selection prior to treatment $[11,12]$. In HNSCC, however, primary RAS mutations are rather uncommon with only $4.6 \%$ of HRAS mutated tumors and their significance for this entity remains unclear $[13,14]$. Just as primary resistance, acquired resistance to cetuximab represents a challenge in the treatment of both mCRC and HNSCC. A recent series of pivotal studies on mCRC suggested that acquired resistance to cetuximab may not only be mediated by selection of rare RAS mutated subclones (from predominantly RAS wildtype tumors) $[15,16]$ but also by acquisition of epitope-modifying EGFR mutations during cetuximab (or panitumumab) treatment [17-19]. In fact, the extracellular domain mutations R451C, S464L, G465R, K467T, I491M and S492R of the EGFR (all located in exon 12) were found in post-therapeutic tumor subclones or antibody-resistant cell lines by next-generation or sanger sequencing. These mutations abrogated antibody binding and, therefore, resulted in clinical resistance to cetuximab and/or panitumumab depending on their localization within the antibody epitopes $[17,19]$.
To investigate if these or related mechanisms may play a role in cetuximab resistance of HNSCC as well, we set out to scan the cetuximab-interacting ectodomain of the EGFR as well as KRAS/NRAS exons 2/3/4 and HRAS exons $2 / 3$ for mutations in a cohort of 46 HNSCC patients by targeted next generation sequencing, 20 of these with available post-cetuximab circulating tumor DNA (ctDNA). We found that RAS mutations can be acquired in a substantial proportion of patients during cetuximab-based treatment and significantly correlate with disease progression. Future studies should quantitatively determine mutational loads that reliably predict the benefit - or lack thereof - from further cetuximab treatment in patients with acquired RAS mutations.

\section{RESULTS}

\section{Patients and treatment}

Of the 46 cetuximab-treated patients, 13 patients $(28 \%)$ were in a curative and 33 patients $(72 \%)$ were in a palliative treatment setting (Table 1). Although 19 of 46 patients $(41 \%)$ had HNSCC of the oropharynx, HPVpositivity was rare with 5 of 46 patients $(11 \%)$. The overall response rate (complete and partial responses) with cetuximab-based treatment was only $47 \%$ implying a high rate of treatment-resistant tumors in this cohort. About two thirds (13/20) of patients with liquid biopsies had progressive disease during combination therapy with Cis or carboplatin, 5-fluorouracil and cetuximab or cetuximab maintenance, respectively. Of the remaining seven patients without progressive disease, two patients refused cetuximab maintenance therapy, one patient died of pneumonia during combination therapy and one patient had severe bleeding complications requiring discontinuation of therapy. The median progression-free survival for patients in the liquid biopsy cohort was 4.9 months (95\% CI 3.4-6.0), the median overall survival 5.2 months (95\% CI 4.0-7.8).

\section{NGS of the cetuximab-interacting EGFR ectodomain and RAS at baseline and in HNSCC cell lines}

We sought to find out i) if tumor subclones expressing a mutated EGFR ectodomain or activating RAS mutations exist in HNSCC tumors before cetuximabbased treatment and ii) if such subclones emerge or expand under the selective pressure of EGFR-directed antibody treatment in this disease. We used NGS to screen EGFR exon 12, KRAS/NRAS exons $2 / 3 / 4$ and HRAS exons $2 / 3$ with a mean number of $>20,000$ reads per exon, ensuring that even rare mutant subclones would be detected (targeted NGS approach schematically shown in Figure 1). 
Table 1: Patient and tumor characteristics with sequencing data of tumor samples.

\begin{tabular}{|c|c|c|c|c|c|c|c|c|c|c|c|c|c|}
\hline \multirow{2}{*}{ Pat. } & \multirow{2}{*}{ Primary site } & \multirow{2}{*}{$\begin{array}{l}\text { HPV- } \\
\text { Status } \\
\text { (p16/HPV- } \\
\text { DNA) }\end{array}$} & \multicolumn{2}{|l|}{$\begin{array}{l}\text { Time of initial } \\
\text { diagnosis }\end{array}$} & \multicolumn{2}{|c|}{ Time of relapse } & \multirow{2}{*}{$\begin{array}{l}\text { Cetuximab } \\
\text { treatment } \\
\text { setting }\end{array}$} & \multirow{2}{*}{$\begin{array}{l}\text { Treatment } \\
\text { combination }\end{array}$} & \multirow{2}{*}{$\begin{array}{l}\text { Origin of } \\
\text { tumor } \\
\text { sample }\end{array}$} & \multirow{2}{*}{$\begin{array}{l}\text { EGFR } \\
\text { exon } \\
12\end{array}$} & \multirow{2}{*}{$\begin{array}{l}\text { KRAS } \\
\text { exon } \\
2 / 3 / 4\end{array}$} & \multirow{2}{*}{$\begin{array}{l}\text { NRAS } \\
\text { exon } \\
2 / 3 / 4\end{array}$} & \multirow{2}{*}{$\begin{array}{l}\text { HRAS } \\
\text { exon } \\
2 / 3\end{array}$} \\
\hline & & & TNM & UICC & TNM & UICC & & & & & & & \\
\hline 1 & Oro-/Hypopharynx & negative $(-/-)$ & cT3 cN2c cMO & IV A & n.a. & n.a. & curative & RT + Cet & intial diagnosis & wt & $w t / w t / w t$ & $w t / w t / w t$ & G13R/wt \\
\hline 2 & Hypopharynx/Larynx & negative $(-/-)$ & cT4a cN2c cM0 & IV A & n.a. & n.a. & curative & TPF; RT + Cet & intial diagnosis & wt & $w t / w t / w t$ & $w t / w t / w t$ & wt/wt \\
\hline 3 & $\begin{array}{l}\text { Oropharynx } \\
\text {. }\end{array}$ & negative $(-/-)$ & pT2 pN2c cM1 & IV C & n.a. & n.a. & palliative & CDDP, 5-FU, Cet & intial diagnosis & wt & $w t / w t / w t$ & $w t / w t / w t$ & $w t / w t$ \\
\hline 4 & Oral Cavity & negative $(-/-)$ & cT4a cN2c cM0 & IV A & n.a. & n.a. & curative & TPF; RT + Cet & intial diagnosis & wt & $w t / w t / w t$ & $w t / w t / w t$ & $w t / w t$ \\
\hline 5 & Oropharynx & negative $(-(-))$ & $\mathrm{cT} 2 \mathrm{cN} 2 \mathrm{~b}$ cMO & IV A & n.a. & n.a. & curative & TPF; RT + Cet & relapse & wt & $w t / w t / w t$ & $w t / w t / w t$ & $w t / w t$ \\
\hline 6 & Oral Cavity & negative $(-/)$ & pT2 pN2b cM0 & IV A & $\mathrm{rCT} 2 \mathrm{cNO} \mathrm{CMO}$ & II & palliative & CDDP, 5-FU, Cet & relapse & wt & $w t / w t / w t$ & $w t / w t / w t$ & $w t / w t$ \\
\hline 7 & Oropharynx & negative $(-/-)$ & cT4a cN2c cM1 & IV C & n.a. & n.a. & palliative & CDDP, 5-FU, Cet & intial diagnosis & wt & $w t / w t / w t$ & $w t / w t / w t$ & $w t / w t$ \\
\hline 8 & Oropharynx & positive $(+/+)$ & сT3 cN2b cMO & IV A & n.a. & n.a. & curative & $\mathrm{RT}+\mathrm{Cet}$ & intial diagnosis & wt & $w t / w t / w t$ & $w t / w t / w t$ & $w t / w t$ \\
\hline 9 & Hypopharynx & negative $(-1+)$ & CT2 cN2c cM0 & IV A & n.a. & n.a. & curative & TPF; RT + Cet & intial diagnosis & wt & $w t / w t / w t$ & $w t / w t / w t$ & $w t / w t$ \\
\hline 10 & Larynx & negative $(-1)$ & CT2 cN3 cM1 & IV C & n.a. & n.a. & palliative & CDDP, 5-FU, Cet & intial diagnosis & wt & $w t / w t / w t$ & $w t / w t / w t$ & $w t / w t$ \\
\hline 11 & Oropharynx & positive $(+/+)$ & pT1 pN2b cM0 & IVA & rCTO cNO cM1 & IV C & palliative & CDDP, 5 -FU, Cet & intial diagnosis & wt & $w t / w t / w t$ & $w t / w t / w t$ & n.e.ln.e. \\
\hline 12 & Oropharynx & negative $(-I-)^{\prime}$ & CT4a cN1 cM0 & IVA & rCT2 CN1 cM0 & III & palliative & Carbo, Taxol, Cet & relapse & wt & $w t / w t / w t$ & $w t / w t / w t$ & n.e./wt \\
\hline 13 & Hypopharynx & negative $(-/)$ & cT4a cN2c cM0 & IV A & n.a. & n.a. & curative & TPF; RT + Cet & relapse & wt & $w t / w t / w t$ & $w t / w t / w t$ & $\mathrm{wt} / \mathrm{wt}$ \\
\hline 14 & Larynx & negative $(-I-)$ & CT2 cNO cMO & II & n.a. & n.a. & curative & TPF; RT + Cet & intial diagnosis & wt & $w t / w t / w t$ & $w t / w t / w t$ & $w t / w t$ \\
\hline 15 & Oral Cavity & negative $(-I-)$ & cT4a cN2c cMO & IV A & n.a. & n.a. & curative & $\mathrm{RT}+\mathrm{Cet}$ & intial diagnosis & wt & $w t / w t / w t$ & $w t / w t / w t$ & $w t / w t$ \\
\hline 16 & Oropharynx & negative $\left(-H_{-}\right)$ & cT4a cN2b cM0 & IV A & $\mathrm{rcT} 4 \mathrm{a}$ cN2b cM0 & IV A & palliative & Carbo, Taxol, Cet & intial diagnosis & wt & $w t / w t / w t$ & $w t / w t / w t$ & $w t / w t$ \\
\hline 17 & Paranasal Sinus & negative $\left.(-)^{-}\right)$ & pT4a pNO cMO & IV A & rCT4a cN2c cM0 & IVA & palliative & CDDP, 5-FU, Cet & intial diagnosis & wt & $w t / w t / w t$ & $w t / w t / w t$ & $w t / w t$ \\
\hline 18 & Oro-/Hypopharynx & negative $(-/-)$ & pT3 pN1 cMO & III & rCT3 cNO cMO & III & palliative & $\mathrm{RT}+\mathrm{Cet}$ & relapse & wt & $w t / w t / w t$ & $w t / w t / w t$ & $w t / w t$ \\
\hline 19 & Oropharynx & negative $(-/-)$ & $\mathrm{CT} \times \mathrm{cN} \times \mathrm{cMO}$ & n.a. & rcT4a cN2b M1 & IV C & palliative & CDDP, 5-FU, Cet & intial diagnosis & wt & $w t / w t / w t$ & $w t / w t / w t$ & $w t / w t$ \\
\hline 20 & Paranasal Sinus & negative $(-/)$ & cT $4 a$ cN2c cM0 & IV A & rCT4a cNO cMO & IV A & palliative & Gem, Vino, Cet & intial diagnosis & wt & $w t / w t / w t$ & $w t / w t / w t$ & $w t / w t$ \\
\hline 21 & Oral Cavity & negative $(-/)$ & cT4a cN2c cM0 & IV A & rcT3 cNO cM1 & IV C & palliative & CDDP, 5-FU, Cet & intial diagnosis & wt & $w t / w t / w t$ & $w t / w t / w t$ & $w t / w t$ \\
\hline 22 & Hypopharynx & negative $(-/-)$ & cT3 cN2b cM0 & IVA & n.a. & n.a. & curative & TPF; RT + Cet & intial diagnosis & wt & $w t / w t / w t$ & $w t / w t / w t$ & $w t / w t$ \\
\hline 23 & Oral Cavity & negative $(-/)$ & cT4a cN2c cM1 & IV C & n.a. & n.a. & palliative & Carbo, Taxol, Cet & intial diagnosis & wt & $w t / w t / w t$ & $w t / w t / w t$ & n.e./n.e. \\
\hline 24 & Oral Cavity & negative $(-H)$ & CT3 cN2c cMO & IV A & ก.a. & n.a. & curative & $\mathrm{RT}+\mathrm{Cet}$ & intial diagnosis & wt & $w t / w t / w t$ & $w t / w t / w t$ & $w t / w t$ \\
\hline 25 & Orophaynx & negative $(-1-)$ & cT4a cN2c cMO & IVA & n.a. & n.a. & curative & TPF; RT + Cet & intial diagnosis & wt & $w t / w t / w t$ & $w t / w t / w t$ & $w t / w t$ \\
\hline 26 & Oropharynx & positive $(+/+)$ & cT4a cN2c cM0 & IV A & n.a. & n.a. & curative & $\mathrm{RT}+\mathrm{Cet}$ & intial diagnosis & wt & $w t / w t / w t$ & $w t / w t / w t$ & $w t / w t$ \\
\hline 27 & Hypopharynx & negative $(-I-)$ & pT2 pN1 cM0 & III & rCT4a cNO cMO & IV A & palliative & CDDP, 5-FU, Cet & relapse & wt & $w t / w t / w t$ & $w t / w t / w t$ & $w t / w t$ \\
\hline 28 & Larynx & negative $(-H)$ & $\mathrm{CT} 2 \mathrm{cNO} \mathrm{cMO}$ & II & $\mathrm{CT} 2 \mathrm{cN} 1 \mathrm{cM} 0$ & III & palliative & CDDP, 5-Fu, Cet & relapse & wt & $w t / w t / w t$ & $w t / w t / w t$ & $w t / w t$ \\
\hline 29 & Hypopharynx & negative $\left.(-)^{-}\right)$ & CT4a cN3 cM1 & IV C & n.a. & n.a. & palliative & CDDP, 5-FU, Cet & intial diagnosis & wt & $w t / w t / w t$ & $w t / w t / w t$ & $w t / w t$ \\
\hline 30 & Oral Cavity & negative $(+/-)$ & PT2 pNO cMO & II & rCT3 CNO CM1 & IV C & palliative & CDDP, 5-FU, Cet & intial diagnosis & wt & $w t / w t / w t$ & $w t / w t / w t$ & G13R/Wt \\
\hline 31 & Oropharynx & negative $(-(-))$ & pT2 pN2b cM0 & IV A & rCTX cN1 cM1 & IV C & palliative & CDDP, 5-FU, Cet & relapse & wt & $w t / w t / w t$ & $w t / w t / w t$ & $w t / w t$ \\
\hline 32 & Oral Cavity & negative $(-H)$ & CT $4 \mathrm{~b}$ c cN3 cM1 & IV B & n.a. & n.a. & palliative & CDDP, 5-Fu, Cet & intial diagnosis & wt & $w t / w t / w t$ & $w t / w t / w t$ & $w t / w t$ \\
\hline 33 & Larynx & negative $(-/-)$ & pT2 pN2b cM0 & IV A & rCT3 $\mathrm{cNO}$ CMO & III & palliative & CDDP, 5-FU, Cet & relapse & wt & $w t / w t / w t$ & $w t / w t / w t$ & $w t / w t$ \\
\hline 34 & Oropharynx & positive $(+/+)$ & рT3 pN2c cM0 & IV A & $\mathrm{rcT} \times \mathrm{CN} \times \mathrm{cM} 1$ & IV C & palliative & CDDP, 5-Fu, Cet & relapse & wt & $w t / w t / w t$ & $w t / w t / w t$ & $w t / w t$ \\
\hline 35 & Hypopharynx & negative $(-1+)$ & CT $3 \mathrm{cN} 2 \mathrm{~b}$ cM0 & IV A & rCTX CN3 cM1 & IV C & palliative & CDDP, 5-FU, Cet & intial diagnosis & wt & $w t / w t / w t$ & $w t / w t / w t$ & $w t / w t$ \\
\hline 36 & Oral Cavity & negative $(-/-)$ & pT2 pN3 cM0 & IV B & $\mathrm{rCT} \times \mathrm{CN} 1 \mathrm{cM} 1$ & IV C & palliative & CDDP, 5-FU, Cet & intial diagnosis & wt & $w t / w t / w t$ & $w t / w t / w t$ & $w t / w t$ \\
\hline 37 & Oral Cavity & negative $(-/-)$ & PT4a pNO cMO & IV A & rCT3 cN1 cM0 & III & palliative & CDDP, 5-FU, Cet & intial diagnosis & wt & $w t / w t / w t$ & $w t / w t / w t$ & $w t / w t$ \\
\hline 38 & Oral Cavity & negative $(-/-)$ & cT4a cN1 cM0 & IVA & rCT3 cN2 cM1 & IV C & palliative & CDDP, 5-FU, Cet & intial diagnosis & wt & $w t / w t / w t$ & $w t / w t / w t$ & $w t / w t$ \\
\hline 39 & Oropharynx & positive $(+++)$ & CT3 CNO cMO & III & CT2 cN1 cM0 & III & palliative & CDDP, 5-FU, Cet & relapse & wt & $w t / w t / w t$ & $w t / w t / w t$ & $w t / w t$ \\
\hline 40 & Hypopharynx & negative $(-/)^{\prime}$ & pT2 pN2c cM0 & IV A & rCT2 cN2c cMO & IV A & palliative & CDDP, 5-FU, Cet & relapse & wt & $w t / w t / w t$ & $w t / w t / w t$ & $w t / w t$ \\
\hline 41 & Oral Cavity & negative $\left.(-)_{-}\right)$ & cT4b cN2c cM1 & IV C & & & palliative & CDDP, 5-FU, Cet & intial diagnosis & wt & $w t / w t / w t$ & $w t / w t / w t$ & n.e./wt \\
\hline 42 & Oropharynx & negative $(-/-)$ & pT2 pN1 cM0 & III & rCTO cNO cM1 & IV C & palliative & CDDP, 5-FU, Cet & intial diagnosis & wt & $w t / w t / w t$ & $w t / w t / w t$ & wt/wt \\
\hline 43 & Hypopharynx & negative $(-1)$ & cT2 cN2b cM1 & IV C & & n.a. & palliative & CDDP, 5-FU, Cet & intial diagnosis & wt & $w t / w t / w t$ & $w t / w t / w t$ & $w t / w t$ \\
\hline 44 & Oropharynx & negative $(-1-)$ & pT1 pN2b cM0 & IV A & rcT2 cNO cM1 & IV C & palliative & CDDP, 5-FU, Cet & intial diagnosis & wt & $w t / w t / w t$ & $w t / w t / w t$ & $w t / w t$ \\
\hline 45 & Oropharynx & negative $(-(-)$ & cT4a cN2c cM0 & IV A & rcT0 cN2c cM1 & IV C & palliative & CDDP, 5-FU, Cet & relapse & wt & $w t / w t / w t$ & $w t / w t / w t$ & $w t / w t$ \\
\hline 46 & Oropharynx & negative $(-1-)$ & CT1 cN3 cMO & IV B & CT4 CNO cMO & IV A & palliative & CDDP, 5-FU, Cet & relapse & wt & $w t / w t / w t$ & $w t / w t / w t$ & $w t / w t$ \\
\hline
\end{tabular}

Abbreviations: HPV human papilloma virus; n.a. not applicable; RT radiotherapy; Cet Cetuximab; TPF Docetaxel/Cisplatin/5-Fluoruracil; CDDP 5-FU Cis-(or Carbo)platin/5Fluoruracil; Carbo, Taxol Carboplatin/Paclitaxel; Gem, Vino Gemcitabine/Vinorelbine; wt wildtype; n.e. not evaluable
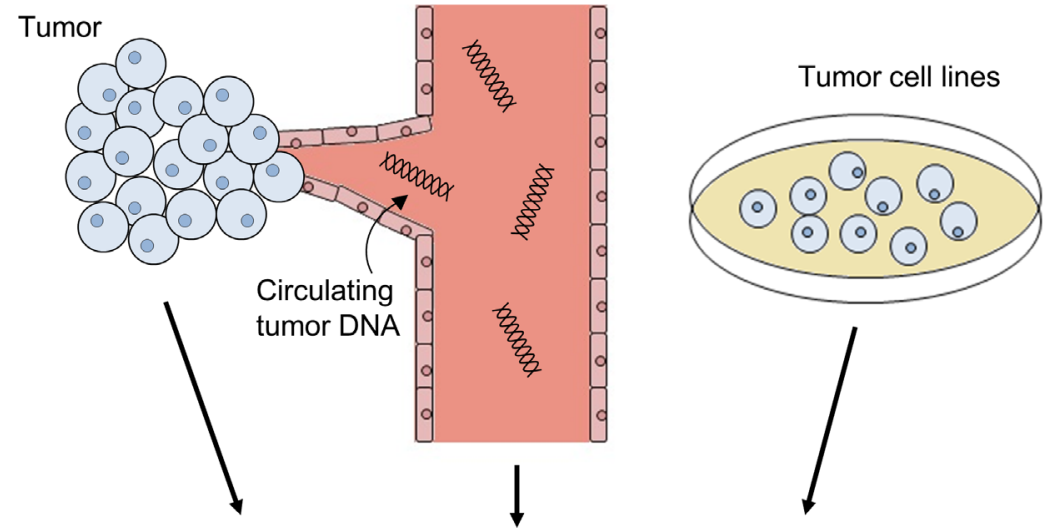

EGFR gene

KRAS gene

NRAS gene
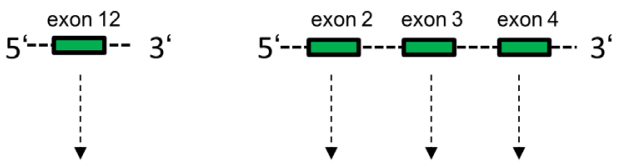

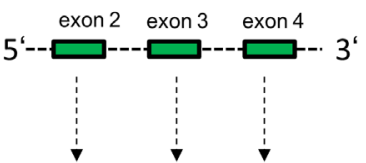

HRAS gene

1st PCR

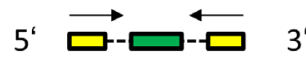

2nd PCR

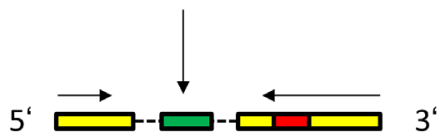

Figure 1: PCR amplification of EGFR and RAS exons for Illumina targeted next generation sequencing. EGFR exon 12, KRAS/NRAS exons 2/3/4 and HRAS exons 2/3 (green) were amplified from tumor tissue of 46 patients, post-cetuximab circulating tumor DNA of 20 patients and from 12 squamous carcinoma cell lines. Illumina-specific sequences for hybridization and sequencing (yellow) as well as patient-specific barcodes (red) were attached in a second PCR step. 
Table 2: Characteristics and sequencing data of squamous cell carcinoma cell lines

\begin{tabular}{|c|c|c|c|c|c|c|c|}
\hline Cellline & Origin & HPV & $\begin{array}{l}\text { EGFR } \\
\text { exon } 12\end{array}$ & $\begin{array}{l}\text { KRAS } \\
\text { exon 2/3/4 }\end{array}$ & $\begin{array}{l}\text { NRAS } \\
\text { exon 2/3/4 }\end{array}$ & $\begin{array}{l}\text { HRAS } \\
\text { exon 2/3 }\end{array}$ & Reference \\
\hline UT-SCC-5 & Tongue & negative & wt & wt/wt/wt & wt/wt/wt & wt/wt & Lin et al. [25] \\
\hline UT-SCC-8 & Supraglotticlarynx & negative & wt & wt/wt/wt & wt/wt/wt & $w t / w t$ & Lin et al. [25] \\
\hline UT-SCC-14 & Tongue & negative & wt & wt/wt/wt & $\mathrm{wt} / \mathrm{wt} / \mathrm{wt}$ & $w t / w t$ & Lin et al. [25] \\
\hline UT-SCC-15 & Tongue & negative & wt & wt/wt/wt & wt/wt/wt & $w t / w t$ & Lin et al. [25] \\
\hline UT-SCC-29 & Glotticlarynx & negative & wt & wt/wt/wt & wt/wt/wt & $w t / w t$ & Lin et al. [25] \\
\hline UT-SCC-42A & Supraglottis & negative & wt & wt/wt/wt & wt/wt/wt & $w t / w t$ & Lin et al. [25] \\
\hline UT-SCC-60A & Tonsil & negative & wt & $w t / w t / w t$ & $w t / w t / w t$ & $w t / w t$ & Lange et al. [22] \\
\hline Cal33 & Tongue & negative & wt & $w t / w t / w t$ & $w t / w t / w t$ & $w t / w t$ & Soffar et al. [24] \\
\hline HSC-4 & Tongue & negative & wt & wt/wt/wt & wt/wt/wt & $w t / w t$ & Lin et al. [25] \\
\hline FaDu & Hypopharynx & negative & wt & wt/wt/wt & wt/wt/wt & $w t / w t$ & Eicheler et al. [26] \\
\hline SAS & Tongue & negative & wt & $w t / w t / w t$ & wt/wt/wt & wt/wt & Soffar et al. [24] \\
\hline SAT & Oral cavity & negative & wt & wt/wt/wt & wt/wt/wt & $w t / w t$ & Nii et al. [23] \\
\hline
\end{tabular}

Abbreviation: wt wildtype

None of the tumor tissue samples of all 46 patients showed evidence of mutations in the cetuximabinteracting EGFR ectodomain or KRAS/NRAS. In line with previous reports, activating HRAS mutations were found in primary tumor samples of two patients $(4.3 \%)$ with one clonal (patient no. 1) and one subclonal mutation (patient no. 30), (Table 1).

All 12 HNSCC cell lines that derived from EGFR antibody-naïve patients were unmutated for EGFR, KRAS/NRAS and HRAS (Table 2).

\section{NGS of the cetuximab-interacting EGFR ectodomain and RAS after cetuximab treatment}

In 20 patients we obtained peripheral blood for ctDNA analysis during and after combination therapy with $\mathrm{Cis}^{-}$or carboplatin, 5-fluorouracil and cetuximab +/cetuximab maintenance (liquid biopsy). Overall, about one third of patients acquired activating RAS mutations in the course of cetuximab-based treatment (KRAS: G12S, G13C; NRAS: Q61K, A146P; HRAS: G13R), while no

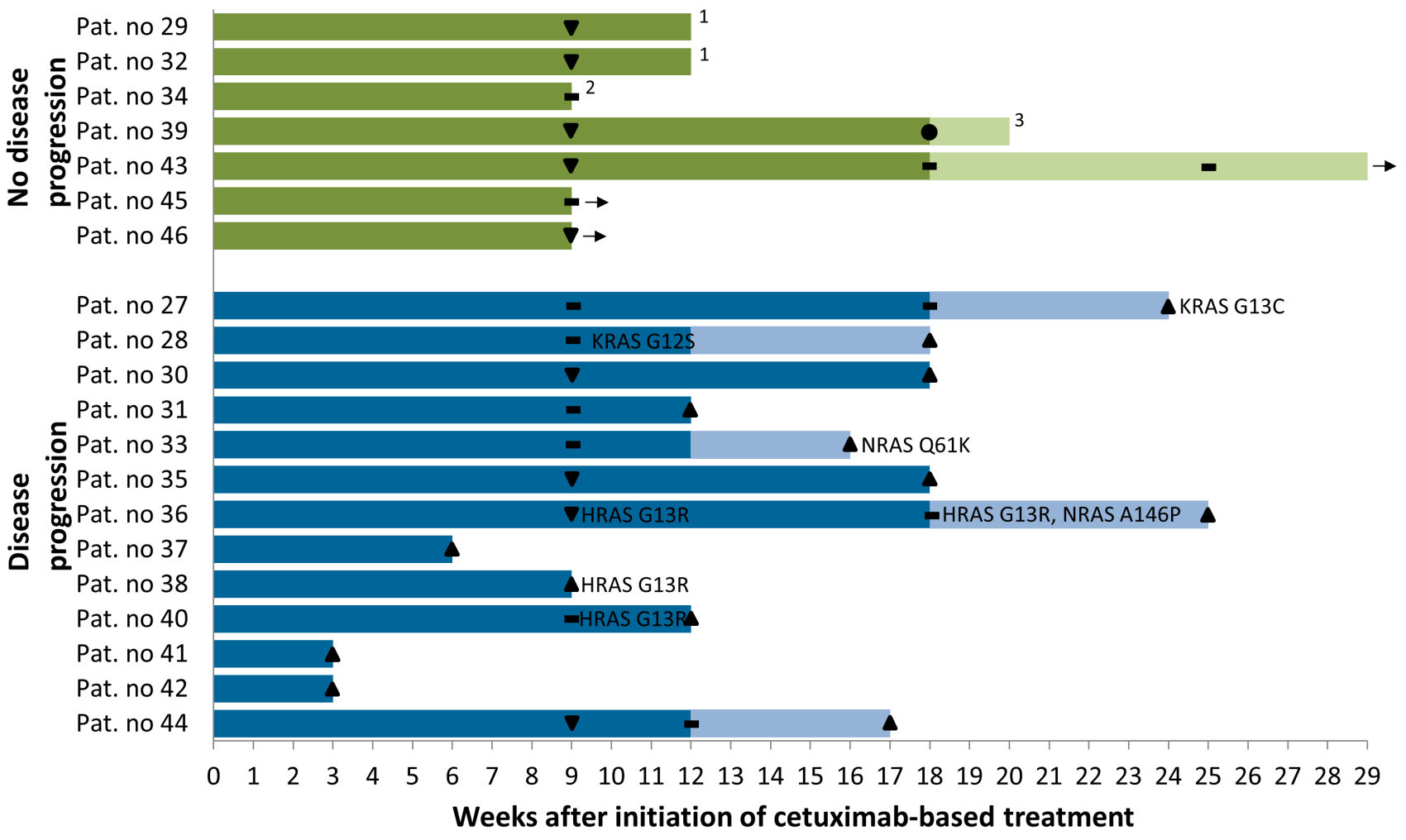

Figure 2: Swimmer plot illustrating treatment, responses and acquired mutations in liquid biopsy cohort of $20 \mathrm{HNSCC}$ patients treated with cetuximab plus chemotherapy. Weeks of combination therapy with cis- or carboplatin, 5-fluorouracil and cetuximab are shown in dark colors, weeks of cetuximab maintenance in light colors. $\bullet$ Complete response, $\boldsymbol{\nabla}$ partial response, - stable disease, $\boldsymbol{\Delta}$ progressive disease. Activating RAS mutations are mapped at the time of their first appearance. ${ }^{1}$ Patients refused further treatment. ${ }^{2}$ Patient died of pneumonia. ${ }^{3}$ Therapy was stopped because of bleeding complications. $\rightarrow$ Ongoing treatment. 
EGFR ectodomain mutations were recorded (Figure $2)$. The emergence of activating RAS clones correlated significantly with disease progression in this cohort (Chisquare, $P=0.032)$. While six of 13 patients (46\%) with progressive disease during cetuximab-based treatment showed evidence of acquired activating RAS mutations, none of the seven responsive patients $(0 \%)$ were mutated for any of the RAS genes at any time point (Figure 2). Some of these mutations appeared early during treatment (earliest detection nine weeks after initiation of cetuximabbased treatment) and preceded clinical progression in half of the patients with the maximum time from mutation detection to clinical progression being 16 weeks in our cohort (Figure 2).

\section{DISCUSSION}

Cetuximab-based treatment is only effective in a subset of patients with HNSCC [7]. However, little is known so far about the molecular mechanisms underlying clinical resistance and we currently lack appropriate biomarkers that could help in identifying patient subsets that are either likely or unlikely to derive benefit from this EGFR-targeted therapy or from prolonged antibody treatment in a cetuximab maintenance setting.

In this study we focused on potential modifications of the EGFR ectodomain that may interfere with antibody binding and activating mutations of RAS, which are known to confer resistance in metastatic colorectal cancer $[10,19]$. While HNSCC tumors are largely negative for RAS mutations at diagnosis $[14,20]$ and EGFR ectodomain mutations have not been detected by conventional sequencing to date, we reasoned that potential resistance-mediating mutations could be present in rare tumor subclones before treatment (undetectable by conventional sequencing) and would subsequently be amplified under the selective pressure of EGFRtargeted antibody treatment. To be able to detect even minor subclones in a background of cells with unmutated EGFR and RAS, we used state-of-the-art targeted NGS technology for highly sensitive and specific identification of mutations in a heterogeneous tumor [21]. By comparing pre- and post-cetuximab genetic material we aimed at uncovering both primary and acquired resistancemediating mutations. Utilizing a sequencing depth that would uncover even rare clones, none of the 46 patients included in this study showed evidence for mutations in EGFR exon 12 or KRAS/NRAS exons 2/3/4 at baseline, while two cases were HRAS mutated. About one third of cases acquired RAS mutations in the course of treatment and, interestingly, all of these cases showed progressive disease while receiving the EGFR antibody. This significant correlation suggests for the first time that activating RAS mutations represent a clinically relevant mechanism of acquired resistance in patients treated with cetuximab.
Two major limitations of this study need to be discussed: First, this study does not formally rule out the (unlikely) possibility that the platinum/5-FU treatment (and not the EGFR-targeted antibody) may induce activating RAS mutations in the HNSCC setting. In the colon cancer setting, however, there is no evidence for the induction of activating RAS mutations by chemotherapy, while there is persuasive evidence for their induction by EGFR-targeting antibodies [15]. Since patients treated with either platinum/5-FU or cetuximab alone are rare, this question is very hard to address. The second limitation of our study refers to the fact that baseline mutational profiling was performed on primary diagnosis tumor tissue (instead of tumor tissue at recurrence) in 7/20 patients and baseline liquid biopsies were not performed. Therefore, we cannot rule out acquisition of mutations between primary diagnosis and recurrence in these seven patients. Given the overall very low RAS mutational frequency in EGFR antibody-naïve patients this point may, however, be of minor clinical relevance.

Taken together, our data suggests that i) RAS mutant subclones can only be found in a minority of HNSCC tumor samples at baseline, but emerge in a substantial proportion of patients during cetuximab treatment, ii) these mutant subclones correlate significantly with disease progression, and iii) may be detectable with state-of-the-art sequencing technology before clinical resistance occurs. Prospectively, determination of such clones may help to tailor anti-EGFR strategies warranting an evaluation in larger prospective clinical trials. More specifically, mutational loads should be defined that reliably predict a lack of response to cetuximab.

\section{METHODS - PATIENTS}

Between October 2012 and January 2016, the Database of the Clinical Cancer Registry of the University Cancer Center Hamburg was screened for HNSCC patients with cetuximab-based treatment. Informed consent was obtained from a total of 46 patients for the use of their diagnostic material (tumor tissue and - in 20 cases - peripheral blood after initiation of cetuximab treatment) as approved by the institutional review board. HPV-Status was part of the routine diagnostic work-up and included a PCR for HPV-DNA and p16 immunohistochemistry. Patients were considered HPV-positive, if both HPVDNA of a high-risk HPV type and overexpression of p16 were present. All other combinations were considered HPV-negative. All tumor samples were validated by a pathologist.

All 20 patients with available post-cetuximab peripheral blood samples were treated with a combination of cetuximab weekly ( $400 \mathrm{mg} / \mathrm{m}^{2}$ as a loading dose, followed by $250 \mathrm{mg} / \mathrm{m}^{2}$ ) and a chemotherapy regimen of cisplatin $\left(100 \mathrm{mg} / \mathrm{m}^{2}\right.$ on day 1$)$ or carboplatin (AUC 5 on day 1) plus 5-fluorouracil (1000 $\mathrm{mg} / \mathrm{m}^{2}$ on days $\left.1-4\right)$ every 
three weeks for a maximum of six courses. Subject to their consent, combination therapy was followed by weekly cetuximab maintenance in patients without progressive disease. Peripheral blood samples for isolation of ctDNA were taken at interim staging after three courses of combination therapy and after completion of combination therapy / maintenance (or at progression if applicable).

\section{MATERIALS AND METHODS}

\section{Cell lines and cell culture}

All 12 squamos cell carcinoma cell lines derived from patients with head and neck tumors [22-25] were cultivated in Dulbecco's Modified Eagle's Medium (Gibco/Life Technologies, Carlsbad, USA) containing $10 \%$ fetal bovine serum (Merck \& Co., Inc., Kenilworth, USA), $4 \mathrm{mM}$ glutamine (Gibco/Life Technologies) and 1\% Penicillin Streptomycin (Gibco/Life Technologies) and were identified using a short tandem repeat multiplex assay (Applied Biosystems/Life Technologies). UTSCC cell lines 5, 8, 14, 15, 29, 42A and 60A were kindly provided by R. Grenman, University of Turku, Finland. The HNSCC p53-negative subline of FaDu (hypopharynx) was obtained from W. Eicheler, University of Dresden, Germany [26], and all other cell lines were kindly provided by M. Baumann, University of Dresden, Germany.

\section{Preparation of genomic DNA from tumor tissue and HNSCC cell lines}

Formalin fixed paraffin embedded tumor tissue was deparaffinized by xylene and ethanol. After digestion with proteinase $\mathrm{K}$ at $56{ }^{\circ} \mathrm{C}$ overnight, genomic DNA was isolated with the QIAamp DNA Micro Kit (Qiagen, Hilden, Germany). Genomic DNA from EGFR positive cell lines was extracted using NucleoSpin Tissue XS kit (Macherey-Nagel, Düren, Germany). Quantity and quality of DNA was evaluated using a Nanodrop spectrophotometer ND-1000 (Thermo Fisher Scientific Inc., Wilmington, USA).

\section{Isolation of circulating tumor DNA (ctDNA) from blood}

Blood samples were centrifuged at $1200 \mathrm{x} \mathrm{g}$ for 10 min within two hours after blood draw. ctDNA was isolated from the serum using the QIAamp Circulating Nucleic Acid Kit (Qiagen).

\section{PCR amplification of EGFR and RAS exons for Illumina targeted next generation sequencing}

In two consecutive PCR reactions, EGFR exon 12, KRAS/NRAS exons 2/3/4 and HRAS exons 2/3 were amplified from tumor or ctDNA and adapters for next-generation sequencing (NGS) were attached (schematically shown in Figure 1). The primers for the first reaction annealed with exon-flanking intron regions (dotted lines) and contained Illumina-compatible adapters (yellow) for later hybridization of amplicons to the Illumina flow cell and for sequencing primer annealing. A second PCR reaction was performed to extend the Illumina adapter sequences and to add a 6 or 7 nucleotide barcode (red) allowing to match each sequence during data analysis to a certain patient / cell line and time point. All primers are shown in Supplementary Table S1.

The PCR was performed using Phusion HS II (Thermo Fisher Scientific Inc.). Amplicons were purified after agarose gel electrophoresis using the NucleoSpin ${ }^{\circledR}$ Gel and PCR Clean-up kit (Macherey-Nagel).

\section{Illumina sequencing and data analysis}

All amplicons were sequenced with a 500-cycle single indexed (8 nucleotides) paired-end run on a MiSeq (Illumina, San Diego, USA). Overlapping paired reads were merged using the software FLASH (v1.2.6) [27]. The format of the merged reads was subsequently converted to FASTA while non-overlapping reads were excluded from further analysis. Usearch (v6.0.307) [28] was employed to dereplicate and cluster the merged reads. Sequences observed less than 30 times were discarded and the remaining sequences were clustered according to their similarity with reference EGFR and RAS exon sequences (Supplementary Table S2). For each cluster of similar sequences, MAFFT (v7.045b) [29] was used to calculate a multiple sequence alignment.

\section{Statistics}

IBM $^{\circledR}$ SPSS $^{\circledR}$ version 22 (IBM, New York, USA) was used for statistical analysis. Contingency tables were calculated and compared using the Pearson Chi-square test. A $p$-value $<0.05$ was considered significant.

\section{ACKNOWLEDGMENTS}

We thank Adam Grundhoff, Malik Alawi and Daniela Indenbirken for assistance with next-generation sequencing and Christopher Ford for proofreading the manuscript. 


\section{CONFLICTS OF INTEREST}

The authors have declared no conflicts of interest.

\section{FUNDING}

This work was supported by Deutsche Forschungsgemeinschaft [BI 1711/1-1 to M.B.]. MB holds a professorship supported by the Hubertus Wald foundation, Hamburg.

\section{Editorial note}

This paper has been accepted based in part on peerreview conducted by another journal and the authors' response and revisions as well as expedited peer-review in Oncotarget.

\section{REFERENCES}

1. Siegel RL, Miller KD, Jemal A. Cancer statistics. CA Cancer J Clin. 2015; 65:5-29.

2. Budach V, Becker ET, Boehmer D, Badakhshi H, Jahn U, Wernecke KD, Stromberger C. Concurrent hyperfractionated accelerated radiotherapy with 5-FU and once weekly cisplatin in locally advanced head and neck cancer. The 10-year results of a prospective phase II trial. Strahlentherapie Onkologie. 2014; 190:250-255.

3. Bonner JA, Harari PM, Giralt J, Cohen RB, Jones CU, Sur RK, Raben D, Baselga J, Spencer SA, Zhu J, Youssoufian H, Rowinsky EK, Ang KK. Radiotherapy plus cetuximab for locoregionally advanced head and neck cancer: 5 -year survival data from a phase 3 randomised trial, and relation between cetuximab-induced rash and survival. Lancet oncology. 2010; 11:21-28.

4. Forastiere AA, Zhang Q, Weber RS, Maor MH, Goepfert H, Pajak TF, Morrison W, Glisson B, Trotti A, Ridge JA, Thorstad W, Wagner H, Ensley JF et al. Long-term results of RTOG 91-11: a comparison of three nonsurgical treatment strategies to preserve the larynx in patients with locally advanced larynx cancer. Journal of clinical oncology. 2013 31:845-852.

5. Posner MR, Hershock DM, Blajman CR, Mickiewicz E, Winquist E, Gorbounova V, Tjulandin S, Shin DM, Cullen K, Ervin TJ, Murphy BA, Raez LE, Cohen RB et al. Cisplatin and fluorouracil alone or with docetaxel in head and neck cancer. The New England journal of medicine. 2007; 357:1705-1715.

6. Vermorken JB, Remenar E, van Herpen C, Gorlia T, Mesia R, Degardin M, Stewart JS, Jelic S, Betka J, Preiss JH, van den Weyngaert D, Awada A, Cupissol D et al. Cisplatin, fluorouracil, and docetaxel in unresectable head and neck cancer. The New England journal of medicine. 2007; 357:1695-1704.
7. Vermorken JB, Mesia R, Rivera F, Remenar E, Kawecki A, Rottey S, Erfan J, Zabolotnyy D, Kienzer HR, Cupissol D, Peyrade F, Benasso M, Vynnychenko I et al. Platinumbased chemotherapy plus cetuximab in head and neck cancer. The New England journal of medicine. 2008; 359:1116-1127.

8. Vermorken JB, Stohlmacher-Williams J, Davidenko I, Licitra L, Winquist E, Villanueva C, Foa P, Rottey S, Skladowski K, Tahara M, Pai VR, Faivre S, Blajman CR et al. Cisplatin and fluorouracil with or without panitumumab in patients with recurrent or metastatic squamous-cell carcinoma of the head and neck (SPECTRUM): an openlabel phase 3 randomised trial. Lancet oncology. 2013; 14:697-710.

9. Bonner JA, Harari PM, Giralt J, Azarnia N, Shin DM, Cohen RB, Jones CU, Sur R, Raben D, Jassem J, Ove R, Kies MS, Baselga J et al. Radiotherapy plus cetuximab for squamous-cell carcinoma of the head and neck. The New England journal of medicine. 2006; 354:567-578.

10. Di Nicolantonio F, Martini M, Molinari F, Sartore-Bianchi A, Arena S, Saletti P, De Dosso S, Mazzucchelli L, Frattini M, Siena S, Bardelli A. Wild-type BRAF is required for response to panitumumab or cetuximab in metastatic colorectal cancer. Journal of clinical oncology. 2008; 26:5705-5712.

11. Lievre A, Bachet JB, Le Corre D, Boige V, Landi B, Emile JF, Cote JF, Tomasic G, Penna C, Ducreux M, Rougier P, Penault-Llorca F, Laurent-Puig P. KRAS mutation status is predictive of response to cetuximab therapy in colorectal cancer. Cancer research. 2006; 66:3992-3995.

12. Lievre A, Laurent-Puig P. Genetics: Predictive value of KRAS mutations in chemoresistant CRC. Nature reviews clinical oncology. 2009; 6:306-307.

13. Wheeler DL, Dunn EF, Harari PM. Understanding resistance to EGFR inhibitors-impact on future treatment strategies. Nature reviews clinical oncology. 2010; 7:493507.

14. Lui VW, Hedberg ML, Li H, Vangara BS, Pendleton K, Zeng Y, Lu Y, Zhang Q, Du Y, Gilbert BR, Freilino M, Sauerwein S, Peyser ND et al. Frequent mutation of the PI3K pathway in head and neck cancer defines predictive biomarkers. Cancer discovery. 2013; 3:761-769.

15. Diaz LA, Jr., Williams RT, Wu J, Kinde I, Hecht JR, Berlin J, Allen B, Bozic I, Reiter JG, Nowak MA, Kinzler KW, Oliner KS, Vogelstein B. The molecular evolution of acquired resistance to targeted EGFR blockade in colorectal cancers. Nature. 2012; 486:537-540.

16. Misale S, Yaeger R, Hobor S, Scala E, Janakiraman M, Liska D, Valtorta E, Schiavo R, Buscarino M, Siravegna G, Bencardino K, Cercek A, Chen CT et al. Emergence of KRAS mutations and acquired resistance to anti-EGFR therapy in colorectal cancer. Nature. 2012; 486:532-536.

17. Arena S, Bellosillo B, Siravegna G, Martinez A, Canadas I, Lazzari L, Ferruz N, Russo M, Misale S, Gonzalez I, Iglesias M, Gavilan E, Corti G et al. Emergence of Multiple 
EGFR Extracellular Mutations during Cetuximab Treatment in Colorectal Cancer. Clinical cancer research. 2015; 21:2157-2166.

18. Montagut C, Dalmases A, Bellosillo B, Crespo M, Pairet S, Iglesias M, Salido M, Gallen M, Marsters S, Tsai SP, Minoche A, Seshagiri S, Serrano S et al. Identification of a mutation in the extracellular domain of the Epidermal Growth Factor Receptor conferring cetuximab resistance in colorectal cancer. Nature medicine. 2012; 18:221-223.

19. Braig F, Marz M, Schieferdecker A, Schulte A, Voigt M, Stein A, Grob T, Alawi M, Indenbirken D, Kriegs M, Engel E, Vanhoefer U, Grundhoff A et al. Epidermal growth factor receptor mutation mediates cross-resistance to panitumumab and cetuximab in gastrointestinal cancer. Oncotarget. 2015; 6:12035-12047. doi: 10.18632/ oncotarget.3574.

20. Bissada E, Abboud O, Abou Chacra Z, Guertin L, Weng X, Nguyen-Tan PF, Tabet JC, Thibaudeau E, Lambert L, Audet ML, Fortin B, Soulieres D. Prevalence of K-RAS Codons 12 and 13 Mutations in Locally Advanced Head and Neck Squamous Cell Carcinoma and Impact on Clinical Outcomes. International journal of otolaryngology. 2013; 2013:848021.

21. Samuel N, Hudson TJ. Translating genomics to the clinic: implications of cancer heterogeneity. Clinical chemistry. 2012; 59:127-137.

22. Lange MJ, Lasiter JC, Misfeldt ML. Toll-like receptors in tonsillar epithelial cells. International journal of pediatric otorhinolaryngology. 2009; 73:613-621.
23. Nii M, Kayada Y, Yoshiga K, Takada K, Okamoto T, Yanagihara K. Suppression of metastasis by tissue inhibitor of metalloproteinase-1 in a newly established human oral squamous cell carcinoma cell line. International journal of oncology. 2000; 16:119-124.

24. Soffar A, Storch K, Aleem E, Cordes N. CDK2 knockdown enhances head and neck cancer cell radiosensitivity. International journal of radiation biology. 2013; 89:523531.

25. Lin CJ, Grandis JR, Carey TE, Gollin SM, Whiteside TL, Koch WM, Ferris RL, Lai SY. Head and neck squamous cell carcinoma cell lines: established models and rationale for selection. Head \& Neck. 2007; 29:163-188.

26. Eicheler W, Zips D, Dorfler A, Grenman R, Baumann M. Splicing mutations in TP53 in human squamous cell carcinoma lines influence immunohistochemical detection. Journal of histochemistry \& cytochemistry. 2002; 50:197204.

27. Magoc T, Salzberg SL. FLASH: fast length adjustment of short reads to improve genome assemblies. Bioinformatics. 2011; 27:2957-2963.

28. Edgar RC. Search and clustering orders of magnitude faster than BLAST. Bioinformatics. 2010; 26:2460-2461.

29. Katoh K, Misawa K, Kuma K, Miyata T. MAFFT: a novel method for rapid multiple sequence alignment based on fast Fourier transform. Nucleic acids research. 2002; 30:30593066. 